\title{
Gestação espontânea em paciente com suspeita de síndrome de Sheehan: relato de caso
}

\section{Spontaneous pregnancy in a patient with suspected Sheehan's syndrome: case report}

\author{
Elvis Runy Paulino da Silva ${ }^{1}$ Cláudio César Monteiro de Castro².
}

1 Médico residente de Clínica Médica, Hospital Universitário Walter Cantídio (HUWC), Universidade Federal do Ceará (UFC), Fortaleza, Ceará, Brasil. 2 Mestrado em Patologia, Professor adjunto do Departamento de Clínica Médica, Universidade Federal do Ceará (UFC), Fortaleza, Ceará, Brasil.

\section{RESUMO}

A Síndrome de Sheehan (SS) é uma das causas mais comuns de hipopituitarismo nos países em desenvolvimento, onde os cuidados obstétricos são mais precários. Decorre da necrose pituitária devido à hemorragia uterina maciça no parto com hipotensão e choque. Pode apresentar um quadro clínico agudo ou crônico, com um hipopituitarismo completo ou parcial. Apesar de rara, a gravidez subsequente pode ser possível se houver uma reserva funcional na produção de gonadotrofinas. Será descrito o caso de uma mulher de 36 anos de idade que descobriu a gestação espontânea durante o período de investigação de uma suspeita de síndrome de Sheehan.

Palavras-chave: Hipopituitarismo. Síndrome de Sheehan. Gestação.

\section{ABSTRACT}

Sheehan syndrome (SS) is one of the most common causes of hypopituitarism in developing countries where obstetric care is more precarious. It results from pituitary necrosis due to massive uterine bleeding at delivery with hypotension and shock. It may present an acute or chronic clinical picture, with complete or partial hypopituitarism. Although rare, subsequent pregnancy may be possible if there is a functional reserve in the production of gonadotrophins. We will describe the case of a 36-year-old woman who discovered spontaneous pregnancy during the investigation period of a suspected Sheehan's syndrome.

Keywords: Hypopituitarism. Sheehan's syndrome. Pregnancy.

Autor correspondente: Elvis Runy Paulino da Silva, Rua Vicente Sales, 481, Cigana, Caucaia, Ceará. CEP: 61605-320. Telefone: +55 85 99644-0421. E-mail: elvisruny@hotmail.com

Conflito de interesses: Não há qualquer conflito de interesses por parte de qualquer um dos autores.

Recebido em: 09 Fev 2018; Revisado em: 29 Mar 2018; Aceito em: 17 Mai 2018. 


\section{INTRODUÇÃO}

A Síndrome de Sheehan (SS) é uma das causas mais comuns de insuficiência pituitária anterior nos países em desenvolvimento, onde os cuidados obstétricos são mais precários. ${ }^{1,2}$ Foi descrita inicialmente em 1937 por H.L. Sheehan como uma insuficiência hipofisária parcial ou completa em decorrência de necrose pós-parto da adenohipófise em mulheres com grave perda sanguínea e hipotensão durante ou logo após o parto. ${ }^{1}$

A prevalência de SS não foi exatamente definida devido ao grande número de pacientes subdiagnosticadas. Em 1965, Sheehan estimou que a prevalência mundial era de 100-200 casos por 100.000 mulheres. ${ }^{2}$ Após sua estimativa, a frequência de SS pode vir diminuindo devido a melhores cuidados obstétricos. No entanto, ainda é alta em regiões pobres do mundo, sendo um problema de saúde comum. ${ }^{3}$

Em 1996, a Organização Mundial de Saúde estimou que 100 mil mulheres estavam morrendo a cada ano devido a SS, e mais de 3 milhões de mulheres no mundo estavam sofrendo de $\mathrm{SS}^{2}$ Na região de Caxemira da Índia, a prevalência de SS encontrado foi de 3,1\% entre as mulheres em 2005, e dois terços das mulheres ainda estavam dando à luz em casa. ${ }^{2}$ $\mathrm{Na}$ Turquia, um estudo recente investigando as causas de hipopituitarismo relatou uma frequência de SS de $27,6 \%$ entre 338 pacientes do sexo feminino, o que colocou a SS como a segunda causa mais comum de hipopituitarismo em mulheres. ${ }^{4}$ Em um estudo retrospectivo das Filipinas em 2010, análise de 82 pacientes do sexo feminino com hipopituitarismo revelou uma prevalência de $14 \%$ secundário a $\mathrm{SS} .{ }^{5}$ No Brasil, não foram encontrados estudos apontando sua prevalência.

A frequência de SS em países desenvolvidos é muito rara se comparado a dos países subdesenvolvidos e em desenvolvimento. ${ }^{6}$ Assim, o diagnóstico de SS pode ser negligenciado e atrasado em muitos anos. ${ }^{3}$ Em 39 pacientes com SS na França, Ramiandrasoa et al. detectaram que o tempo médio de atraso ao diagnóstico, ou seja, o tempo entre o parto e diagnóstico, foi de 9,1 anos. $^{2}$ Os motivos apontados para essa demora foram os sinais e sintomas inespecíficos e a raridade da SS, o que faz com que seja pouca reconhecida entre muitos profissionais de saúde.

A gestação em pacientes após necrose pituitária é muito rara. Foram encontradas as descrições de alguns relatos de casos de pacientes que apresentaram gestações subsequentes à $\mathrm{SS}$, seja durante tratamento com reposição hormonal, com indução de ovulação ou de forma espontânea. ${ }^{7-9} \mathrm{O}$ objetivo desse artigo é descrever um relato de caso raro, alertando para a necessidade de uma melhor assistência às parturientes como forma de prevenção e sobre o reconhecimento precoce e tratamento da SS. Relatamos o caso de uma mulher de 36 anos de idade que descobriu a gestação espontânea durante o período de investigação de anemia e, concomitantemente, de uma suspeita de síndrome de Sheehan.

\section{RELATO DE CASO}

Paciente do sexo feminino, 36 anos, foi referenciada de uma
Unidade Básica de Saúde a um ambulatório especializado do Hospital Universitário Walter Cantídio em 2013 devido uma anemia a esclarecer. Apresentava quadro clínico de astenia, fadiga, sensação de dispneia progressiva aos esforços, tontura, cefaleia intermitente de leve a moderada intensidade iniciados há dois anos, além de palidez. Na história ginecológica e obstétrica pregressa a paciente teve 3 gestações, 3 partos e nenhum aborto, com menarca aos 15 anos, ciclos menstruais regulares até última gestação em 2010, que evoluiu com óbito fetal seguido de hemorragia importante durante o parto cesáreo com necessidade de hemotransfusão. Após último parto, evoluiu com oligomenorreia, astenia, lipotimia, dispneia aos esforços, labilidade emocional e constipação. Estava em amenorreia há seis meses e referia desconforto em hipogastro. Durante investigação diagnóstica, evidenciou-se TSH e T4-L sugestivos de hipotireoidismo central secundário à provável síndrome de Sheehan. Foi diagnosticada também gestação, mesmo antes do início do tratamento com reposição dos hormônios deficientes. A Tabela 1 mostra um resumo dos exames realizados.

Tabela 1. Resultado de exames laboratoriais.

\begin{tabular}{lll}
\hline Exames & Resultados & $\begin{array}{l}\text { Valores de referência do } \\
\text { laboratório }\end{array}$ \\
\hline $\mathrm{Hb}$ & $8,7 \mathrm{~g} / \mathrm{dL}$ & $11,5-16 \mathrm{~g} / \mathrm{dL}$ \\
$\mathrm{VCM} / \mathrm{HCM}$ & $93 \mathrm{u} 3 / 29 \mathrm{pg}$ & $80-98 \mathrm{u} 3 / 26,5-33,5 \mathrm{pg}$ \\
$\begin{array}{l}\text { Indice Saturação } \\
\text { da Transferrina }\end{array}$ & $47 \%$ & $15-50 \%$ \\
$\mathrm{TSH}$ & $1,43 \mathrm{mIU} / \mathrm{ml}$ & $0.34-5,6 \mathrm{mIU} / \mathrm{ml}$ \\
$\mathrm{T} 4-\mathrm{L}$ & $0,20 \mathrm{ng} / \mathrm{dL}$ & $0,54-1,24 \mathrm{ng} / \mathrm{dL}$ \\
Cortisol basal & $2,5 \mu \mathrm{g} / \mathrm{dL}$ & $5,5-30 \mu \mathrm{g} / \mathrm{dL}$ \\
\hline
\end{tabular}

\section{DISCUSSÃO}

Embora a etiopatogênese da SS ainda não esteja totalmente compreendida, duas condições básicas são bem estabelecidas para o desenvolvimento da necrose pituitária: o aumento da hipófise no período gestacional, geralmente não acompanhado do aumento do suprimento sanguíneo, juntamente com a hemorragia uterina maciça no parto. ${ }^{1,2}$ Além disso, outros fatores contribuintes, como o menor tamanho da sela túrcica em alguns casos, o estado de hipercoagulabilidade, fatores genéticos e vasoespasmo também podem estar envolvidos no processo. ${ }^{2,3,6}$ Apesar de achados controversos em alguns estudos, existem evidências que apontam para um componente de autoimunidade envolvido na evolução da síndrome, pois o hipopituitarismo parcial tende a evoluir com o tempo. Uma hipótese é que a necrose do tecido hipofisário expõe antígenos que ativam o sistema imunológico, produzindo anticorpos antipituitários que levam à destruição das células remanescentes. ${ }^{2,3}$

Para o diagnóstico da Síndrome de Sheehan é importante uma história obstétrica cuidadosa. São comuns relatos de trabalho de parto difícil, com hemorragia maciça durante ou logo após 
o parto, evoluindo posteriormente com agalactia e amenorreia secundária. ${ }^{10}$ Estão presentes sinais clínicos agudos ou crônicos de hipopituitarismo e sela vazia ou parcialmente vazia à ressonância de sela túrcica. ${ }^{1}$ Em pacientes com tal história médica e quadro clínico, a avaliação de níveis hormonais basais, incluindo prolactina, T4 livre, TSH, ACTH, cortisol, FSH, LH, estradiol e IGF-1, podem ser suficientes para o diagnóstico de síndrome de Sheehan. ${ }^{1}$ Porém, outras podem necessitar de testes dinâmicos da função hipofisária.

A função pituitária pode ser parcialmente preservada em alguns casos para os níveis de LH e FSH na Síndrome de Sheehan. ${ }^{6}$ Mesmo havendo uma frequente reserva funcional de gonadotrofinas, as mulheres com esta síndrome raramente menstruam espontaneamente, muitas vezes necessitando do uso de citrato de clomifeno para induzir a ovulação. ${ }^{8}$ Embora rara, a gravidez pode ocorrer sem tratamento em pacientes com produção suficiente de gonadotrofinas. ${ }^{6}$ Cerca de 20 gravidezes em 13 pacientes foram relatadas nos últimos 20 anos. ${ }^{8}$

Pacientes com a Síndrome de Sheehan e hipotireoidismo central podem apresentar os níveis séricos de TSH diminuídos, normais ou aumentados. Este TSH tem grau de sinalização elevado, bioatividade intrínseca reduzida e taxa metabólica de clearence diminuída. ${ }^{11}$ A evolução do hipotireoidismo após a necrose hipofisária é de forma lenta, com a redução gradual do TSH e logo após do T4L, que gera estímulo à síntese e à secreção do TSH nos tireotrofos remanescentes sobre os núcleos hipotalâmicos paraventriculares. Os baixos níveis de

\section{REFERÊNCIAS}

1. Soares DV, Conceição FL, Vaisman, M. Aspectos diagnósticos e terapêuticos da síndrome de Sheehan. Arq Bras Endrocrinol Metab. 2008;52(5):23-31.

2. Diri H, Karaca Z, Tanriverdi F, Unluhizarci K, Kelestimur F. Sheehan's syndrome: new insights into an old disease. Endocrine. 2016;51(1):22-31.

3. Kilicli F, Dokmetas HS, Acibucu F. Sheehan's syndrome. Gynecol Endocrinol. 2013;29(4):292-5.

4. Tanriverdi F, Dokmetas HS, Kebapci N, Kilicli F, Atmaca $\mathrm{H}$, Yarman S, et al. Etiology of hypopituitarism in tertiary care institutions in Turkish population: analysis of 773 patients from pituitary study group database. Endocrine. 2014;47(1):198-205.

5. Elumir-Mamba LA, Andag-Silva AA, Fonte JS, Mercado-Asis LB. Clinical profile and etiology of hypopituitarism at the University of Santo Tomas Hospital. Philipp J Intern Med. 2010;48:23-27.

6. Shivaprasad C. Sheehan's syndrome: newer advances. Indian J Endocrinol Metab. 2011;15(Suppl 3):S203-7. cortisol e a redução do tônus hipotalâmico de somatostatina, devido à queda na secreção de $\mathrm{GH}$, podem contribuir para o aumento de TSH nestas pacientes. ${ }^{11}$

A instituição de uma terapia de substituição hormonal adequada durante a gravidez subsequente à síndrome é imprescindível, uma vez que o hipopituitarismo não tratado está associado a alta morbimortalidade fetal e materna. ${ }^{8}$ A reposição hormonal não deve ser iniciada antes dos glicocorticoides, a menos que resultados laboratoriais precisos mostrem um eixo hipotalâmico-hipófise-adrenal normal. São utilizados níveis de T3 e T4 em vez de níveis de TSH para avaliar as doses de substituição de tiroxina, além da melhora clínica., ${ }^{2,3}$

\section{CONCLUSÃO}

A Síndrome de Sheehan é uma patologia com apresentação clínica variável a depender do grau de acometimento da adenohipófise e que pode levar a repercussões importantes à saúde da mulher. Apesar de sua prevalência vir diminuindo devido à melhora dos cuidados obstétricos, ela ainda é frequente em países subdesenvolvidos e em desenvolvimento. O diagnóstico ainda é tardio na maioria dos casos, devido sua evolução lenta, apresentação clínica variada e o pouco reconhecimento da síndrome por profissionais. A SS nem sempre é completa, podendo haver perda seletiva da função da hipófise anterior. Portanto, a gravidez subsequente, apesar de ser evento extremamente raro, pode ocorrer se houver uma reserva funcional na produção de gonadotrofinas.

7. Zargar AH, Masoodi SR, Laway BA, Sofi FA, Wani AI. Pregnancy in Sheehan's syndrome: a report of three cases. J Assoc Physicians India. 1998;46(5):476-8.

8. Algün E, Ayaktaa H, Harmanb M, Topala C, Aksoya H. Spontaneous pregnancy in a patient with Sheehan's syndrome. Eur J Obstet Gynecol Reprod Biol. 2003;110(2):242-4.

9. See TT, Lee SP, Chen HF. Spontaneous pregnancy and partial recovery of pituitary function in a patient with Sheehan's syndrome. J Chin Med Assoc. 2005;68(4):187-90.

10. Mary H, Samuels MD. Sheehan's Syndrome. Endocrinologist. 2004;12(1):25-29.

11. Oliveira JH, Persani L. Investigating the paradox of hypothyroidism and increased serum thyrotropin (TSH) levels in Sheehan's syndrome: characterization of TSH carbohydrate content and bioactivity. J Clin Endocrinol Metab. 2001;86:1694-9.

\section{Como citar:}

Silva ER, Castro CC. Gestação espontânea em paciente com suspeita de síndrome de Sheehan: relato de caso. Rev Med UFC. 2018 out-dez;58(4):71-73. 